
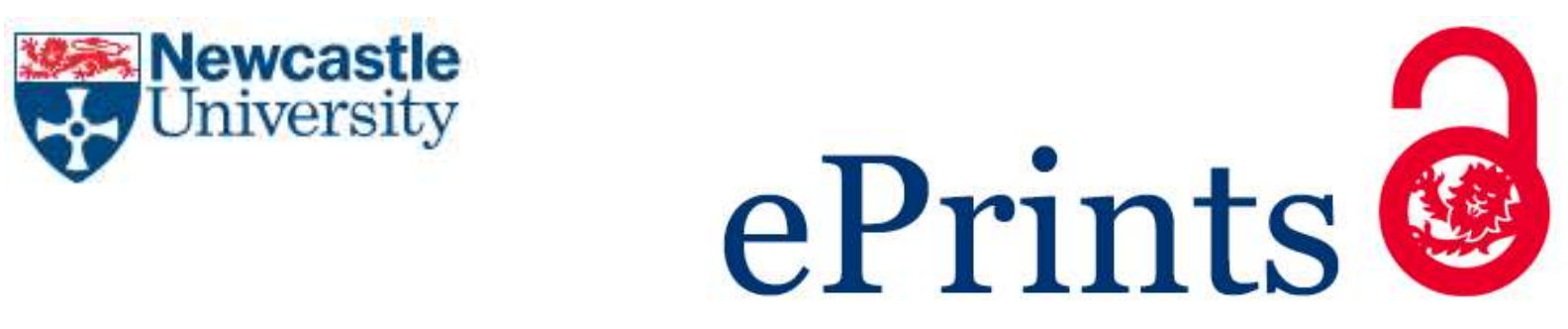

Prufert F, Bonengel S, Kollner S, Griesser J, Wilcox MD, Chater PI, Pearson JP, Bernkop-Schnurch A. $\zeta$ potential changing nanoparticles as cystic fibrosis transmembrane conductance regulator gene delivery system: An in vitro evaluation. Nanomedicine 2017, 12(22), 2713-2724.

\title{
Copyright:
}

This is the authors' accepted manuscript of an article that has been published in its final definitive form by Future Medicine, 2017

DOI link to article:

https://doi.org/10.2217/nnm-2017-0115

Date deposited:

$11 / 01 / 2018$

Embargo release date:

29 September 2018 
Nanomedicine (Lond). 2017 Nov;12(22):2713-2724. doi: 10.2217/nnm-20170115. Epub 2017 Sep 29.

$\zeta$ potential changing nanoparticles as cystic fibrosis transmembrane conductance regulator gene delivery system: an in vitro evaluation. 


\section{$\zeta$ potential changing nanoparticles as cystic fibrosis transmembrane conductance regulator gene delivery system: an in vitro evaluation}

Felix Prüfert ${ }^{1}$, Sonja Bonengel ${ }^{1}$, Saskia Köllner ${ }^{2}$, Janine Griesser ${ }^{2}$, Matthew D Wilcox ${ }^{3}$, Peter I Chater $^{3}$, Jeffrey P Pearson ${ }^{3} \&$ Andreas Bernkop-Schnürch ${ }^{1 *}$

${ }^{1}$ Department of Pharmaceutical Technology, Institute of Pharmacy, University of Innsbruck, Innrain 80/82, 6020 Innsbruck, Austria

2 ThioMatrix GmbH, Research Center Innsbruck, Trientlgasse 65, 6020 Innsbruck, Austria.

${ }^{3}$ Institute for Cell and Molecular Biosciences, Medical School, Newcastle University, Newcastle upon Tyne, NE2 4HH, United Kingdom

*Author for correspondence: Tel.: +43-512- 507 58601; Fax: +43-512- 507 58699; e-mail: andreas.bernkop@uibk.ac.at 


\section{Abstract}

\section{Aim of the study}

Aim of the study was the development of $\zeta$ potential changing nanoparticles as gene delivery system for the cystic fibrosis transmembrane conductance regulator gene. Methods

Chitosan and carboxymethyl cellulose were modified with phosphotyrosine, a substrate for the brush border enzyme alkaline phosphatase. With these synthesized derivatives, different nanoparticle formulations, including the cystic fibrosis transmembrane conductance regulator gene were prepared by ionic gelation.

\section{Results}

A change from negative to positive $\zeta$ potential after enzymatic cleavage could be observed. Transfection studies with HEK-293 and Caco-2 cells showed transfection rates comparable to Lipofectamine 2000. Transfection efficiencies were significantly decreased when phosphate cleavage and thus $\zeta$ potential change was inhibited by phosphatase inhibitor.

\section{Conclusion}

The developed nanoparticles represent a promising gene delivery system. 


\section{Keywords}

$\zeta$ potential changing nanoparticles

gene delivery

cystic fibrosis transmembrane conductance regulator

phosphotyrosine

phosphatase 


\section{Introduction}

With ever-growing interest in gene therapy, the demand for efficient gene delivery systems increases rapidly. The introduction of gene therapeutics into cells allows for the replacement of defect or missing genes and hence the expression of certain proteins. Because of their high selectivity and target-specificity they are supposed to show a higher efficiency and less side effects compared to conventional drugs [1]. However, efficient gene delivery still remains a major challenge in pharmaceutical technology as low cellular uptake, enzymatic DNAdegradation and the mucosal barrier require efficient gene delivery systems, especially in case of mucosal drug delivery. As viral vectors show high immunogenicity, research concentrated on non-viral delivery systems in the last years. Most systems consist of cationic polymers and lipids $[1,2]$ as they easily form complexes with DNA and it has been shown that positively charged nanoparticles show a higher rate of cell uptake than negatively charged ones [3-5]. However, most epithelial cells are covered by the mucus gel layer, which, due to its negative net charge immobilizes positive particles before they reach the epithelium. In contrast, negatively charged nanoparticles diffuse significantly faster in mucus [6]. Recent studies have shown that $\zeta$ potential changing nanoparticles can combine these two desirable but contrary properties of positive and negative $\zeta$ potential7 [7-10]. These nanoparticles bear negatively charged functional groups that are substrates for brush border enzymes. Once the nanoparticles have crossed the mucus layer, these functional groups will be cleaved off enzymatically, changing the $\zeta$ potential of the particles. Thus, they are immobilized and can be preferably endocytosed by epithelial cells. For this study, phosphotyrosine, a substrate for intestinal alkaline phosphatase (IAP), was chosen as functional group and conjugated with each chitosan and carboxymethyl cellulose as described before [7]. The modified polymers were then used to prepare nanoparticles containing pDNA. The pDNA chosen for the present 
study includes a CFTR gene, which encodes for the cystic fibrosis transmembrane conductance regulator, an ion channel that transports chloride ions from the cell interior to the mucus gel layer, keeping the osmotic homeostasis. Genetic defects in the CFTR gene cause dysregulation of the fluid transport across the epithelium, thus leading to mucus thickening mainly in the respiratory and gastrointestinal tract and resulting in cystic fibrosis (CF). To date, cystic fibrosis, the most common life-threatening autosomal recessive disease among Caucasians with an incidence of 1 in 2500 [11-13], is mainly treated symptomatically. The only causal treatments are Ivacaftor (licensed in 2012), which is targeting a specific mutation related to approximately $5 \%$ of CF patients [13], and Lumacaftor in combination with Ivacaftor. On the contrary, CFTR gene therapy could be used for causal treatment of CF patients regardless of the mutation [13-15].

The pDNA containing nanoparticles were investigated regarding their suitability as gene delivery systems, using cultured epithelial cells as a model system. Therefore, the $\zeta$ potential and the change of the $\zeta$ potential after enzymatic cleavage was investigated. HEK-293 and Caco-2 cells were used to evaluate the cytotoxic potential of the nanoparticles and the phosphate cleavage by the monolayers. Moreover, the transfection efficiency of the nanoparticles was evaluated with both cell lines. The protective effect of the nanoparticles against enzymatic degradation of the pDNA by DNase I was investigated using gel electrophoresis. 


\section{Materials and methods}

\subsection{Materials}

Phosphotyrosine was purchased from Bachem Holding AG, Bubendorf, Switzerland. 1-Ethyl-3-(3-dimethylaminopropyl) carbodiimide hydrochloride was obtained from Acros, Geel, Belgium. Midori Green Advance was obtained from Biozym Biotech Trading GmbH, Vienna, Austria. Nuclease-free, DEPC treated water was purchased from LACTAN GmbH, Graz, Austria. EndoFree ${ }^{\circledR}$ plasmid purification kit was obtained from Qiagen, Hilden, Germany. Lipofectamine ${ }^{\circledR} 2000$ Transfection Reagent and goat serum (10\%) were purchased from Fisher Scientific - Austria GmbH, Vienna, Austria. Rabbit monoclonal to alkaline phosphatase, tissue non-specific and goat anti-rabbit IgG H\&L; Alexa Fluor ${ }^{\circledR}$ were purchased from Abcam, United Kingdom. Cell culture microplates were purchased from Greiner Bio-One GmbH, Kremsmünster, Austria. All other cell culture supplies were purchased at Biochrom $\mathrm{GmbH}$, Berlin, Germany. Chitosan (20 kDA) was purchased from Heppe Medical Chitosan GmbH, Germany. Carboxmethyl cellulose $(90 \mathrm{kDa})$, alkaline phosphatase from bovine intestinal mucosa, phosphatase inhibitor cocktail 2 and all other substances were purchased from Sigma-Aldrich Handels GmbH, Vienna, Austria.

\subsection{Polymer synthesis $\&$ characterization}

Phosphotyrosine (PTyr) was conjugated to each chitosan (CS) and carboxymethyl cellulose (CMC) in carbodiimide-catalyzed reactions as described previously [7]. For the synthesis of the

phosphotyrosine-bearing chitosan derivative (CS-PTyr), $1 \mathrm{~g}$ of 1-ethyl-3-(3-dimethylaminopropyl) carbodiimide hydrochloride (EDAC) was added to $50 \mathrm{~mL}$ of an aqueous solution of $250 \mathrm{mg}$ phosphotyrosine and $500 \mathrm{mg}$ chitosan (20 kDA) and the $\mathrm{pH}$ was adjusted to 5 . After an overnight reaction at $40{ }^{\circ} \mathrm{C}$ the mixture was purified from unbound 
PTyr and EDAC by dialysis for 3 days against $12 \mathrm{~L}$ of demineralized water using Zellutrans/Roth dialysis membrane (MWCO $3500 \mathrm{Da}$ ). The dialysis medium was exchanged every $12 \mathrm{~h}$. The purified product was lyophilized using a Gamma 1-16 LSC (Christ, Osterode, Germany) and stored dry at $8^{\circ} \mathrm{C}$.

In a similar manner, phosphotyrosine was conjugated with CMC. $1 \mathrm{~g}$ of CMC (90kDa) in $50 \mathrm{~mL}$ demineralized water was activated by addition of $2 \mathrm{~g}$ EDAC at a pH of 5 . After an incubation time of $15 \mathrm{~min}, 500 \mathrm{mg}$ of PTyr was added and the mixture was stirred over night at $40{ }^{\circ} \mathrm{C}$. The synthesized polymer was purified as described above using $\mathrm{Nadir}^{\circledR}$ cellulose membrane (MWCO 10-20 kDa), lyophilized and stored at $8^{\circ} \mathrm{C}$. As controls, the syntheses were performed without EDAC.

The amount of conjugated PTyr was quantified spectrophotometrically. Samples of the synthesized derivatives were dissolved in $0.5 \mathrm{M} \mathrm{HCl}$ (CS-PTyr) or $20 \mathrm{mM}$ TRIS buffer (pH 8.5) (CMC-PTyr), respectively, and the absorbance at $265 \mathrm{~nm}$ was determined. The amount of conjugated PTyr was then calculated using a calibration curve of PTyr standards.

\subsection{Preparation of the plasmid}

CFTR DNA subcloned into the pIRES2-EGFP vector (Clontech, Palo Alto, CA, USA) as described by Gong et al. [16] was a gift from Prof. Paul Linsdell. pIRES-EGFP-CFTR plasmid DNA was amplified in E. coli cells and purified using an EndoFree ${ }^{\circledR}$ Plasmid Purification Kit from Qiagen. After purification, the plasmid was dissolved in nuclease free water and quantified with a NanoDrop 2000c UV-Vis Spectrophotometer (Thermo Fisher Scientific, Wilmington, DE, USA). Aliquots were stored at $-20^{\circ} \mathrm{C}$ until further use. 


\subsection{Nanoparticle preparation \& characterization}

Nanoparticles were prepared by ionic gelation utilizing the opposite charges of the CS-PTyr and the pDNA and accordingly the CMC-Ptyr. The formulations were composed of either CS-PTyr with pDNA or CS-Tyr with pDNA and CMC-PTyr, respectively. Therefore, $0.1 \%(\mathrm{~m} / \mathrm{v})$ stock solutions of both polymers dissolved in $25 \mathrm{mM}$ sodium acetate were prepared and $\mathrm{pH}$ was adjusted to 6.8 . These solutions were sterile filtered through $0.2 \mu \mathrm{m}$ filters. Aliquots of the polymer solutions and the pDNA solution were heated separately to $55^{\circ} \mathrm{C}$. CS-PTyr was then diluted in preheated nuclease free water and mixed with either pDNA or pDNA and CMC-PTyr. Each mixture was vortexed immediately for $20 \mathrm{~s}$. Different formulations were prepared and compositions of the different formulations are given in Table 1.

The $\zeta$ potential of these nanoparticles was measured using a Nicomp ${ }^{\mathrm{TM}} 380$ ZLS (Santa Barbara, CA, USA) with a laser wavelength of $632.8 \mathrm{~nm}$ and the E-Field Strength set to $5 \mathrm{~V} / \mathrm{cm}$. To determine the change of $\zeta$ potential after enzymatic cleavage it was measured before and after addition of two units of IAP.

\subsection{Encapsulation efficiency}

The encapsulation efficiency of the pDNA in the nanoparticles was determined within a gel electrophoresis study. Nanoparticles were prepared as described above and investigated by gel electrophoresis using a HE 33 mini submarine electrophoresis unit (Hoefer, Inc., San Francisco, CA, USA). Naked pDNA served as control. An 1\% agarose gel was prestained with Midori Green Advance DNA stain (Biozym Biotech Trading GmbH, Vienna, Austria) and DNA bands were detected under UV-light. 


\subsection{Cytotoxicity}

To detect cytotoxic effects of the polymers and nanoparticles on HEK-293 and Caco-2 cells a MTT (3-[4,5-dimethylthiazol-2-yl]-2,5-diphenyl tetrazolium bromide) assay was performed [17]. Viable cells reduce MTT to insoluble formazan crystals, which can be quantified spectrophotometrically once redissolved in DMSO. Therefore, the HEK-293 and Caco-2 cells, respectively, were each seeded in 96 -well plates at a concentration of $2 \times 10^{5}$ cells $/ \mathrm{mL}$ of MEM (minimum essential medium, containing $10 \%$ fetal bovine serum and antibiotics). Cells were then incubated for $24 \mathrm{~h}$ at $37{ }^{\circ} \mathrm{C}$ and $5 \% \mathrm{CO}_{2}$ and afterwards washed twice with phosphate buffered saline (PBS). Subsequently, $100 \mu \mathrm{l}$ of the samples with corresponding final pDNA concentrations of $5-20 \mu \mathrm{g} / \mathrm{mL}$ were applied. Pure MEM and Triton $x^{\circledR} 1004 \%$ (v/v) were applied as negative and positive controls. The cells were incubated for 4.5 hours under abovementioned conditions before discarding the supernatant and washing the cells twice with PBS. To detect the amount of viable cells $100 \mu \mathrm{l}$ of a $0.5 \%(\mathrm{~m} / \mathrm{v})$ MTT solution in MEM was added and incubated for 4.5 hours before removing the supernatant. The precipitated formazan crystals were dissolved in $100 \mu \mathrm{l}$ of DMSO and the absorbance of the formazan solutions was measured at a wavelength of $570 \mathrm{~nm}$ with a reference wavelength of $690 \mathrm{~nm}$ using a microplate reader (Tecan infinite, M200 spectrometer, Grödig, Austria). The cell-viability was calculated as percentage of the negative control.

\subsection{Enzymatic phosphate cleavage by HEK-293 \& Caco-2 monolayers}

The enzymatic cleavage of phosphate off the nanoparticles by HEK-293 and Caco-2 monolayers was investigated. Cells were seeded in 24 -well plates in a concentration of $2 \times 10^{5}$ cells/mL. After incubating for $24 \mathrm{~h}$, cells were washed twice with a solution consisting of 268 mM glucose and 27 mM HEPES ( $\mathrm{pH}$ 7.4) before adding $500 \mu \mathrm{l}$ of the nanoparticle suspensions. These were added with and without the presence of phosphatase inhibitor cocktail $2(1 \% \mathrm{v} / \mathrm{v})$. 
The cells were then incubated at $37{ }^{\circ} \mathrm{C}$ and $5 \% \mathrm{CO}_{2}$. Samples of $50 \mu \mathrm{l}$ were taken at predetermined time points and the enzymatic reaction was terminated immediately by adding $5 \mu \mathrm{l}$ of $\mathrm{H}_{2} \mathrm{SO}_{4}(3.6 \mathrm{M})$. The amount of cleaved phosphate was quantified utilizing the malachite green assay as described previously [8]. $100 \mu \mathrm{l}$ of malachite green reagent ( $6 \mathrm{~mL}$ of a $7.5 \%$ ammonium molybdate solution $+10 \mathrm{~mL}$ of a $0.15 \%$ malachite green solution in sulfuric acid $(3.6 \mathrm{M})+0.4 \mathrm{~mL}$ of a $11 \%$ Triton-X 100 solution) were added to each sample and the absorbance at a wavelength of $630 \mathrm{~nm}$ was measured. The amount of released phosphate was calculated using a calibration curve of phosphate standards.

In order to confirm that the cell lines contain the alkaline phosphatase, immunocytochemistry images were taken from the stained metallo-enzyme alkaline phosphatase (AP) in Caco-2 and HEK293 cells according to the vendor protocol (Abcam; UK). Both cell lines were cultured on a 96 well plate in a density of $2 * 10^{5}$ cells $/ \mathrm{ml}$ in $100 \mu$ of minimum essential medium (MEM) for $24 \mathrm{~h}$ at $37{ }^{\circ} \mathrm{C}$ and $5 \% \mathrm{CO}_{2}$. First, the cells were fixed with $100 \mu \mathrm{l}$ of pure methanol and incubated for $5 \mathrm{~min}$ at room temperature. In order to obtain permeable cells, $100 \mu \mathrm{l} 0.1 \%$ $(\mathrm{m} / \mathrm{m})$ Triton X-100 diluted in phosphate buffer saline (PBS) buffer $\mathrm{pH} 7.4$ were added and incubated for $5 \mathrm{~min}$ at room temperature. Thereafter, cells were treated with $50 \mu \mathrm{l}$ normal goat serum (10\%) (Fisher Scientific, UK) for $1 \mathrm{~h}$ at $4^{\circ} \mathrm{C}$ to block the unspecific interactions. In addition, half of the cell vials were incubated with $50 \mu \mathrm{l}$ primary antibody to AP (Rabbit monoclonal to alkaline phosphatase, tissue non-specific; Abcam; UK) in a concentration of $4.88 \mu \mathrm{g} / \mathrm{ml}$ as positive control (stored in PBS buffer containing $0.01 \%(\mathrm{~m} / \mathrm{m}$ ) sodium azide, $50 \%(\mathrm{~m} / \mathrm{m})$ glycerol and $0.05 \%(\mathrm{~m} / \mathrm{m})$ bovine serum albumin). In contrast, the other half was treated with $50 \mu \mathrm{l}$ PBS buffer $\mathrm{pH} 7.4$ as negative control. The plate was then incubated at $4^{\circ} \mathrm{C}$ for $12 \mathrm{~h}$. After three washing steps with PBS buffer, $50 \mu \mathrm{l}$ of secondary antibody (Goat antirabbit IgG H\&L; Alexa Fluor ${ }^{\circledR}$ ) in a concentration on $2 \mu \mathrm{g} / \mathrm{ml}$ (stored in PBS buffer containing 
$0.02 \%(\mathrm{~m} / \mathrm{m})$ sodium azide, $30 \%(\mathrm{~m} / \mathrm{m})$ glycerol and $1 \%(\mathrm{~m} / \mathrm{m})$ bovine serum albumin) were applied to all cells and incubated for $1 \mathrm{~h}$ at $4^{\circ} \mathrm{C}$. Thereafter, all cells were once again washed for three times washed with PBS buffer. Furthermore, between all experimental steps PBS buffer $\mathrm{pH} 7.4$ was utilized as washing buffer. Images were taken with a combination of a microscope (Motic Type AE31, Motic GmbH, Germany) and a camera (ProgRes, Jenoptik, Germany) utilizing ProgRes CapturePro software (Jenoptik, Germany) to visualize fluorescence.

\subsection{Transfection studies}

Transfection studies were performed with HEK-293 and Caco-2 cells. Cells were seeded in 24-well plates in a concentration of $4 \times 10^{5}$ cells per $\mathrm{mL}$ and incubated for $48 \mathrm{~h}$ to gain approximately $90 \%$ confluency prior to transfection. The nanoparticle formulations NP-CS 1 and NP-CMC-CS, prepared as described above were diluted with OPTI MEM $^{\circledR}$ reduced serum medium without antibiotics to a final concentration of $5 \mu \mathrm{g} \mathrm{pDNA} / \mathrm{mL}$. Each well was loaded with $500 \mu \mathrm{L}$ of the samples. As control, wells were loaded with samples containing the nanoparticles and the phosphatase inhibitor as well as samples containing naked DNA and pDNA-Lipofectamine ${ }^{\circledR} 2000$ complex, respectively. The pDNA-Lipofectamine complex was prepared according to the instructions of the manufacturer in a ratio of $1: 2$ (DNA:Lipofectamine $^{\mathrm{TM}}$ 2000) and showed a $\zeta$ potential of $14.7 \pm 1.42$ and a size of 190.65 \pm 92.09 . The pDNA concentration applied to the cells corresponded to that of the nanoparticle formulations. After an incubation time of $4.5 \mathrm{~h}$ the supernatant was discarded and $500 \mu \mathrm{l}$ of MEM with FCS and antibiotics was added. After $48 \mathrm{~h}$, the supernatant was discarded and cells were harvested for the GFP assay [18]. 


\subsection{Green fluorescence protein assay}

The transfection efficiency was determined by a GFP assay as described previously [18]. The cells were washed twice with pre-warmed PBS before lysing them with $100 \mu \mathrm{l}$ of PBS containing $0.2 \%$ Triton X-100 and $1 \mathrm{mM}$ dithiothreitol, followed by a freeze-thaw cycle. Afterwards, $50 \mu \mathrm{l}$ of each lysate was transferred to a 96 -well fluorescence plate to determine the amount of GFP by measuring the fluorescence at an excitation wavelength of $488 \mathrm{~nm}$ and an emission wavelength of $510 \mathrm{~nm}$ with a microplate reader (Tecan infinite, M200 spectrometer, Grödig, Austria).

\subsection{Statistical data analysis}

GraphPad Prism ${ }^{\circledR} 5$ was used for statistical data analysis. One-way ANOVA with Bonferroni post test was performed. Mean values were calculated from at least three independent samples, respectively. 


\section{Results}

\subsection{Characterization of polymers \& nanoparticles}

The synthesis of CS-PTyr and CMC-PTyr and the formation of the nanoparticles is shown schematically in Figure 1. The derivatization of the polymers was accomplished by a covalent amide bond formation catalyzed by EDAC. In the case of CS-PTyr, the bond was formed between the amino group of the CS and the carboxyl group of PTyr while with CMC-PTyr it was established between the carboxyl group of the CMC and the amino group of PTyr. The amount of conjugated PTyr was determined by spectrophotometric analysis, showing coupling rates of $862.34 \pm 30.68 \mu \mathrm{mol} / \mathrm{g}$ chitosan (0.139 PTyr subunits / monomer subunit CS) and $258.54 \pm 12.92 \mu \mathrm{mol} / \mathrm{g} \mathrm{CMC} \mathrm{(0.062} \mathrm{PTyr} \mathrm{subunits} \mathrm{/} \mathrm{monomer} \mathrm{subunit} \mathrm{CMC).} \mathrm{The} \mathrm{control}$ syntheses without EDAC showed only negligible amounts of PTyr.

\subsection{Nanoparticles}

The derivatized polymers and the pDNA were used to prepare nanoparticles via ionic gelation. The nanoparticles formed spontaneously after mixing due to the ionic interactions of CS-PTyr with pDNA and CMC-PTyr. Different formulations were prepared, consisting of either CS-PTyr and pDNA in different ratios (\#1-3) or CS-PTyr and both pDNA and CMC-PTyr (\#4). The $\zeta$ potential of these formulations before and after incubation with IAP was investigated by electrophoretic light scattering. As illustrated in Figure 2, a $\zeta$ potential change after enzymatic cleavage was measured in all tested formulations. A $\zeta$ potential change from negative to positive was observed for the formulations \#1 and \#4. Thus, these two formulations were selected for further investigations. 


\subsection{Encapsulation efficiency}

The successful encapsulation of the pDNA into the nanoparticles was verified via gel electrophoresis showing a strong fluorescence signal of stained pDNA on the bottom of the application pocket in case of nanoparticles being unable to permeate into the gel. Merely in case of formulation \#1 a minor amount of pDNA was obviously leaking out of the nanoparticles migrating into the gel. In contrast, naked pDNA moved in the gel showing bands being characteristic for a plasmid (Figure 3).

\subsection{Cytotoxic effects}

Cell viability studies were performed to investigate a possible cytotoxic effect of the formulations on each HEK-293 and Caco-2 cells. The cells were incubated with different concentrations of the formulations chosen for the transfection studies. The incubation time was set to $4.5 \mathrm{~h}$ in accordance with the transfection protocol. The cell viability was then quantified with a MTT assay. As shown in Figure 4, a concentration dependent cytotoxicity was observed. While the lowest concentration did not show any cytotoxic effect at all, the higher concentrations affected the cells. A cell viability of approximately $75 \%$ was measured after treatment with the highest concentration. For control reasons, also the formulations with Lipofectamine ${ }^{\circledR} 2000$ and the phosphatase inhibitor cocktail were investigated in the concentration chosen for further investigations, showing no cytotoxic effect.

\subsection{Enzymatic phosphate cleavage on HEK-293 \& Caco-2 monolayers}

The time dependent release of phosphate from the nanoparticles after enzymatic cleavage by HEK-293 and Caco-2 monolayers, respectively, was evaluated and results are shown in Figure 5. On both cell lines, released phosphate could be determined. However, for Caco-2 cells a higher amount of cleaved phosphate was measured than for HEK-293 cells. In both cases, the 
extent of released phosphate was slightly higher for the formulation \#4 compared to \#1. The phosphate-cleavage was significantly reduced in the presence of the phosphatase inhibitor. As illustrated in Figure 6, the enzyme alkaline phosphatase could be determined in both tested cell lines. Due to the fluorescence in pictures $A$ and the absence of fluorescence in pictures $B$, a successful binding of the primary antibody to the enzyme and a subsequent binding of the fluorescent secondary antibody and thus the presence of alkaline phosphatase was proven.

\subsection{Transfection studies}

GFP assays were performed to verify successful transfection of each HEK-293 and Caco-2 cells with nanoparticle formulations \#1 and \#4, respectively. To investigate the influence of the $\zeta$ potential change induced by enzymatic phosphate cleavage on the transfection efficiency the samples were applied to the cells without and with phosphatase inhibitor. As shown in, Figure 7, a significantly increased transfection efficiency for both formulations compared to naked pDNA could be observed in both cell lines. Furthermore, the transfection efficiency was comparable or higher (formulation \#4) than that of the pDNA-Lipofectamine ${ }^{\circledR} 2000$ complex, which was applied as control. In contrast, when applied in presence of the phosphatase inhibitor, the transfection efficiency was in the same range as the naked pDNA. With HEK-293 cells, the absolute transfection efficiencies are higher and the differences between the samples and the controls are more pronounced than with the Caco-2 cells. 


\section{Discussion}

The CFTR gene therapy is a promising strategy for the treatment of cystic fibrosis as it could provide causal treatment independent of the mutation and thus be applicable for a large number of patients [14]. However, as with all gene drugs, this demands for an efficient gene delivery system because several biological barriers have to be overcome. This system should protect the gene from enzymatic degradation, deliver it to the epithelia by crossing the mucus gel layer and increase the cellular uptake. In order to fulfill these requirements the combination of contrary properties is needed, as for an efficient diffusion through the mucus gel layer negatively charged particles are advantageous [6], while cellular uptake is increased by positively charged particles [4]. In recent studies, nanoparticles were developed, that are capable of changing their $\zeta$-potential in a controlled manner after enzymatic cleavage by brush border enzymes [7-10]. -In the present study, this strategy was used to develop a nanoparticulate CFTR gene delivery system and evaluate the improvement of transfection efficiency caused by the $\zeta$ potential change.

Therefore, two different polymers were modified with phosphotyrosine as functional group as described before by our group [7]. Chitosan and carboxymethyl cellulose were each conjugated with PTyr in EDAC mediated reactions forming covalent bonds between the amino group and the carboxyl group of the respective substrates (Figure 1). PTyr was chosen, as it is substrate for the membrane-bound enzyme alkaline phosphatase. The nanoparticles were prepared via ionic gelation. Two different types of nanoparticles were prepared in different formulations. Type 1 consisted of CS-PTyr and pDNA, in which the negatively charged pDNA worked as the counter ion for the positive amino groups of the chitosan. Type 2 contained the pDNA and both polymers CS-PTyr and CMC-PTyr, in which both, pDNA and CMC-PTyr worked as counter ion. Formulations with different ratios were prepared (Table 1) and for the resulting 
nanoparticles, $\zeta$ potentials between -7 and $-17 \mathrm{mV}$ could be determined (Figure 2). Thus, all four formulations exhibited the required negative $\zeta$ potential. In the next step, the change of $\zeta$ potential after incubation with intestinal alkaline phosphatase (IAP) was evaluated. The enzyme hydrolyses the phosphate ester and the remaining tyrosine is neutral compared to the negatively charged phosphotyrosine. This results in a reduced amount of negatively charged groups displayed by the nanoparticles and the ratio between positive and negative groups displayed by the nanoparticles shifts towards the positive ones, resulting in a $\zeta$ potential shift. A shift of $\zeta$ potential could be observed for all formulations, and a change to positive $\zeta$ potential was detected for formulations \#1 and \#4. The difference between the $\zeta$ potential before and after enzymatic cleavage was higher for formulation \#4 consisting of both CS-PTyr and CMC-PTyr as more PTyr groups are available for cleavage in this formulation. According to these results, formulations \#1 and \#4 were chosen for further investigations.

The chosen nanoparticle formulations were tested for their encapsulation efficiency. Encapsulation of the pDNA was higher in formulation \#4 than in formulation \#1, as in case of the latter some pDNA was released into the gel. This indicates that the ionic gelation was more effective in presence of CMC-PTyr with its carboxyl group and the pDNA is entrapped by the polymers during the nanoparticle formation. This is in accordance with other reports that ternary polymeric nanoparticles show improved properties regarding DNA encapsulation and gene delivery compared to binary complexes [19-21]. Furthermore, the incomplete encapsulation of formulation \#1 could explain the increasing negative $\zeta$ potential with increasing DNA concentrations as observed for formulations \#1, \#2 and \#3.

While both polymers are well-known for their low toxicity and classified as safe substances by the FDA, cytotoxicity studies were performed to evaluate the cytotoxic profile of the nanoparticles and investigate suitable nanoparticle concentrations for the upcoming studies 
employing HEK-293 and Caco-2 cells. A slight concentration dependent toxicity was detected and hence the lowest concentrations corresponding to $5 \mu \mathrm{g} p \mathrm{pA} / \mathrm{mL}$ were chosen for further investigations, as they showed no decrease in cell viability at all.

For a successful transfection, the change of $\zeta$ potential is the crucial factor as positively charged particles enhance the cellular uptake [4]. In case of the prepared nanoparticles, this $\zeta$ potential change is achieved by the enzymatic hydrolysis of the phosphate group of the phosphotyrosine and thus this enzymatic cleavage is the essential requirement for transfection studies. Consequently, the enzymatic phosphate cleavage by HEK-293 and Caco-2 monolayers was evaluated. Both cell lines are reported to produce phosphatase $[22,23]$. The presence of the enzyme in both cell lines could be confirmed by immunocytochemistry. While a phosphate release was detected with both cell lines, the amount of released phosphate was higher when incubated on Caco-2 cells, which is most likely due to the lower activity of alkaline phosphatase in HEK-293 cells [23]. To assess the impact of the enzymatic cleavage and thus the $\zeta$ potential change, the phosphate elimination was also evaluated in presence of phosphatase inhibitor. When the inhibitor was applied, the phosphate release was significantly inhibited. A complete suppression could not be achieved in the applied recommended concentration, which is in accordance with the information of the supplier, that a complete suppression is not possible with one inhibitor cocktail due to different isoforms [24]. On the other hand, a higher concentration of inhibitor might cause cytotoxic effects.

The cellular uptake of the nanoparticles was investigated by transfection studies with both, HEK-293 and Caco-2 cells. The CFTR gene was subcloned on a vector that also contains a gene encoding for green fluorescence protein (GFP). The expression of the GFP by the cells after incubation with the nanoparticles was analyzed to evaluate the transfection efficiency. Cells were transfected by both investigated formulations, while for formulation \#4, containing both 
CS-PTyr and CMC-PTyr, higher transfection rates were detected than for formulation \#1. Transfection efficiencies were significantly higher than that of naked pDNA and comparable to the pDNA-Lipofectamine ${ }^{\circledast} 2000$ complex. Transfection rates and differences were higher for HEK-293 cells compared to Caco-2 cells. HEK-293 cells are generally regarded to be easier transfected, which also explains why transfection of HEK-293 cells is higher despite the lower activity of alkaline phosphatase. Generally, the transfection efficacy of the pDNA-Lipofectamine ${ }^{\circledR} 2000$ complex was in this study comparatively low, as in most other studies Lipofectamine ${ }^{\circledR} 2000$ leads to log-fold improvements. Detailed dose response studies, however, were not subject of this investigation.

The higher transfection of formulation \#4 compared to formulation \#2 reflect the different encapsulation efficiencies and again supports the findings about enhanced gene delivery capabilities of ternary compared to binary nanoparticle formulations [19]. Furthermore, these results are in accordance with the determination that the $\zeta$ potential of formulation $\# 4$ after enzymatic cleavage was higher than that of formulation \#1, and thus facilitating cell uptake. Electrostatic interactions between the negatively charged cell membrane and positively charged particles promote the cell-uptake and higher charge leads to increased cell-uptake [3]. In contrast, inhibition of the enzymatic cleavage and hence prevention of $\zeta$ potential change effected a significantly decreased transfection efficiency which was in the range of naked pDNA. Consequently, the $\zeta$ potential change is decisive for an effective cellular uptake of the developed nanoparticles. 


\section{Conclusion}

This is, to our knowledge, the first time, that pDNA with a cystic fibrosis transmembrane conductance regulator gene was incorporated in $\zeta$ potential changing nanoparticles. A change of $\zeta$ potential from negative to positive, induced by enzymatic cleavage of the phosphotyrosine, was detected. The pDNA was successfully encapsulated and a successful transfection of HEK-293 and Caco-2 cells, with a transfection efficiency in the range of Lipofectamine 2000, was shown. According to these findings, the developed nanoparticles seem to be a promising gene delivery system. 


\section{Summary points}

- Phosphotyrosine was conjugated to chitosan and carboxymethyl cellulose, respectively.

- pDNA containing a cystic fibrosis transmembrane conductance regulator gene was successfully incorporated into nanoparticles.

- The $\zeta$ potential of the nanoparticles changed from negative to positive after enzymatic cleavage by phosphatase.

- The conjugated phosphotyrosine is enzymatically cleaved by HEK-293 and Caco-2 monolayers.

- The encapsulated pDNA was protected against enzymatic degradation by DNase I.

- HEK-293 and Caco-2 cells were successfully transfected.

- Transfection efficiency was comparable to that of the pDNA-Lipofectamine ${ }^{\circledR} 2000$ complex and significantly higher compared to naked pDNA.

- Change of $\zeta$ potential was decisive for effective cellular uptake. 


\section{References}

1 Patil SD, Rhodes DG, Burgess DJ. DNA-based therapeutics and DNA delivery systems: A comprehensive review. In: AAPS J. (2005) E61-77.

2 Pack DW, Hoffman AS, Pun S, Stayton PS. Design and development of polymers for gene delivery. Nat Rev Drug Discov, 4(7), 581-593 (2005).

3 Salatin S, Maleki Dizaj S, Yari Khosroushahi A. Effect of the surface modification, size, and shape on cellular uptake of nanoparticles. Cell Biology International, 39(8), 881890 (2017).

4 Alexis F, Pridgen E, Molnar LK, Farokhzad OC. Factors Affecting the Clearance and Biodistribution of Polymeric Nanoparticles. Molecular Pharmaceutics, 5(4), 505-515 (2008).

5 Adler AF, Leong KW. Emerging links between surface nanotechnology and endocytosis: impact on nonviral gene delivery. Nano today, 5(6), 553-569 (2010).

6 Crater JS, Carrier RL. Barrier properties of gastrointestinal mucus to nanoparticle transport. Macromol Biosci, 10(12), 1473-1483 (2010).

7 Perera G, Zipser M, Bonengel S, Salvenmoser W, Bernkop-Schnürch A. Development of phosphorylated nanoparticles as $\zeta$ potential inverting systems. European Journal of Pharmaceutics and Biopharmaceutics, 97, Part A, 250-256 (2015).

8 Bonengel S, Prüfert F, Perera G, Schauer J, Bernkop-Schnürch A. Polyethylene imine-6phosphogluconic acid nanoparticles - a novel $\zeta$ potential changing system. International Journal of Pharmaceutics, 483(1-2), 19-25 (2015).

9 Bonengel $S$, Prüfert $F$, Jelkmann $M$, Bernkop-Schnürch $A$. $\zeta$ potential changing phosphorylated nanocomplexes for pDNA delivery. International Journal of Pharmaceutics, 504(1-2), 117-124 (2016).

10 Suchaoin W, Pereira de Sousa I, Netsomboon K, Lam HT, Laffleur F, Bernkop-Schnürch A. Development and in vitro evaluation of $\zeta$ potential changing self-emulsifying drug delivery systems for enhanced mucus permeation. International Journal of Pharmaceutics, 510(1), 255-262 (2016).

11 Mishra A, Greaves R, Massie J. The Relevance of Sweat Testing for the Diagnosis of Cystic Fibrosis in the Genomic Era. The Clinical biochemist. Reviews / Australian Association of Clinical Biochemists., 26(4), 135-153 (2005).

12 Tobias ES, Connor M, Ferguson-Smith M. Essential medical genetics (John Wiley \& Sons, 2011).

13 O'Reilly R, Elphick HE. Development, clinical utility, and place of ivacaftor in the treatment of cystic fibrosis. Drug Design, Development and Therapy, 7, 929-937 (2013).

14 Burney TJ, Davies JC. Gene therapy for the treatment of cystic fibrosis. The Application of Clinical Genetics, 5, 29-36 (2012).

15 Alton EWFW, Armstrong DK, Ashby D et al. Repeated nebulisation of non-viral CFTR gene therapy in patients with cystic fibrosis: a randomised, double-blind, placebocontrolled, phase 2b trial. The Lancet Respiratory Medicine, 3(9), 684-691 (2015). 
16 Gong X, Burbridge SM, Cowley EA, Linsdell P. Molecular determinants of $\mathrm{Au}(\mathrm{CN})(2)(-)$ binding and permeability within the cystic fibrosis transmembrane conductance regulator $\mathrm{Cl}(-)$ channel pore. The Journal of Physiology, 540(Pt 1), 39-47 (2002).

17 Plumb JA. Cell sensitivity assays : the MTT assay. Methods Mol Med, 28, 25-30 (1999).

18 Hauptstein S, Prüfert F, Bernkop-Schnürch A. Self-nanoemulsifying drug delivery systems as novel approach for pDNA drug delivery. Int J Pharm, 487(1-2), 25-31 (2015).

19 Yang Z, Jiang Z, Cao Z et al. Multifunctional non-viral gene vectors with enhanced stability, improved cellular and nuclear uptake capability, and increased transfection efficiency. Nanoscale, 6(17), 10193-10206 (2014).

20 Li J, Chen Q, Zha Z et al. Ternary polyplex micelles with PEG shells and intermediate barrier to complexed DNA cores for efficient systemic gene delivery. Journal of Controlled Release, 209, 77-87 (2015).

21 Lai TC, Kataoka K, Kwon GS. Bioreducible polyether-based pDNA ternary polyplexes: Balancing particle stability and transfection efficiency. Colloids and Surfaces B: Biointerfaces, 99, 27-37 (2012).

22 Jumarie C, Malo C. Alkaline phosphatase and peptidase activities in Caco-2 cells: Differential response to triiodothyronine. In Vitro Cellular \& Developmental Biology Animal, 30(11), 753-760 (1994).

23 Lenz W, Herten M, Gerzer R, Drummer C. Regulation of natriuretic peptide (urodilatin) release in a human kidney cell line. Kidney Int, 55 (1999) 91-99.

24 Sigma Aldrich: Gleiser I, Yaish P, Barnea-Gedalyahu E, Zharhary D. Phosphatase Inhibitor Cocktail. http://www.sigmaaldrich.com/technicaldocuments/articles/biowire/phosphatase-inhibitor.html 


\section{Financial \& competing interests disclosure}

The research leading to these results has received funding from the European Community's Seventh Framework Programme (FP7/2007-2013) under grant agreement number 280761. The authors have no other relevant affiliations or financial involvement with any organization or entity with a financial interest in or financial conflict with the subject matter or materials discussed in the manuscript apart from those disclosed.

No writing assistance was utilized in the production of this manuscript. 


\section{Figures}

\section{Figure 1.}

CS

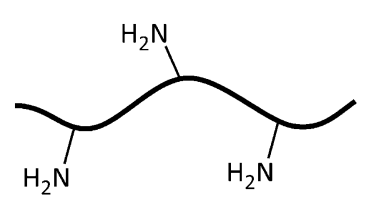

+ EDAC $\downarrow$ PTyr

CS-PTyr

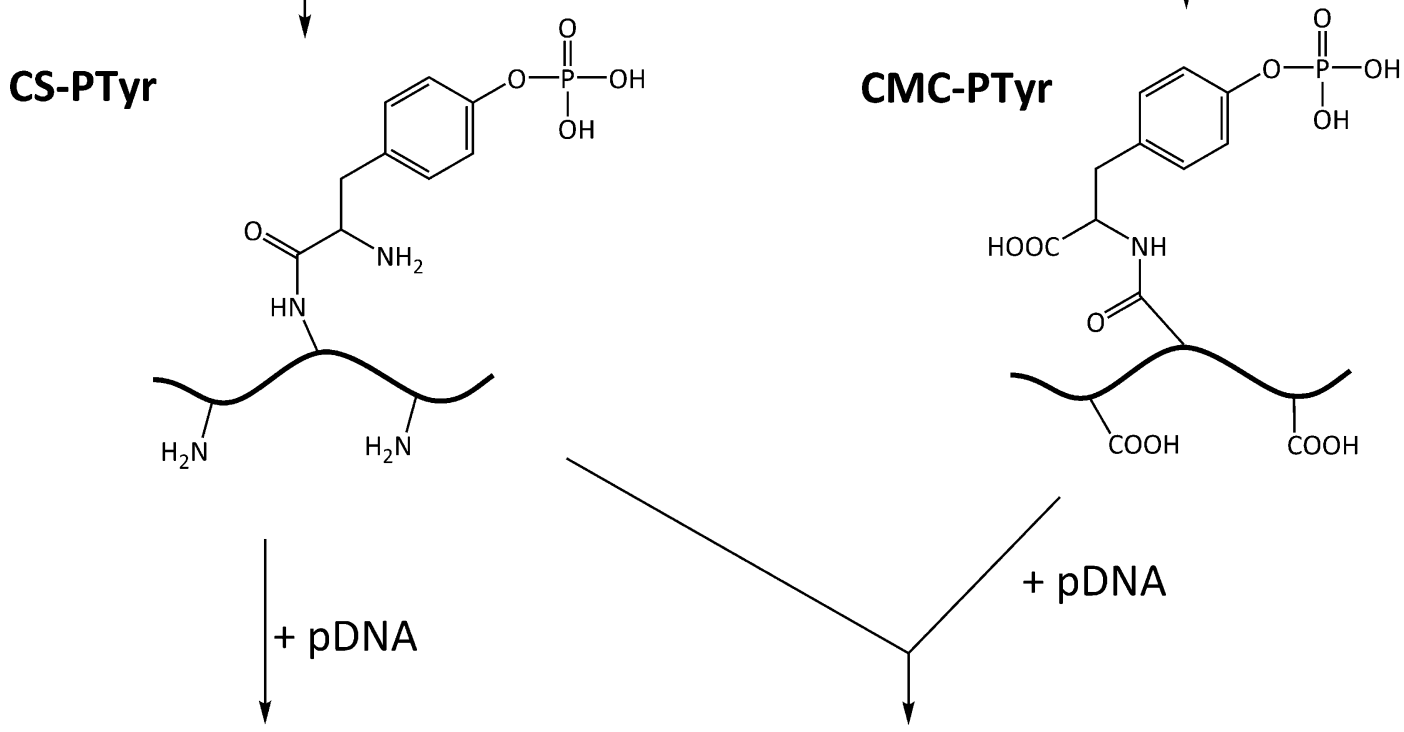

CMC

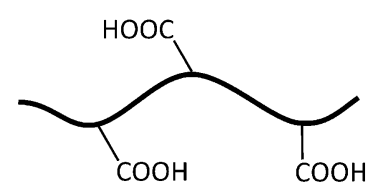

+ EDAC $\downarrow+$ PTyr

A

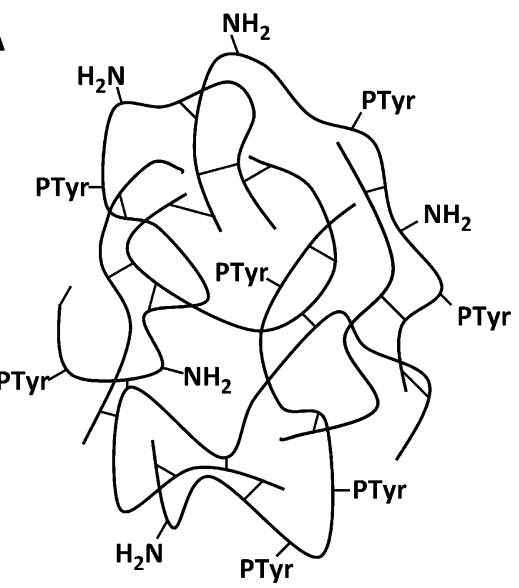

Scheme of synthesis and nanoparticle formulation: Phosphotyrosine (PTyr) was conjugated with each chitosan (CS) and carboxymethyl cellulose (CMC) in EDAC mediated reactions. Nanoparticles were prepared by ionic gelation of CS-PTyr with pDNA (A) or CS-PTyr with both CMC-Ptyr and pDNA (B), respectively. 
Figure 2.

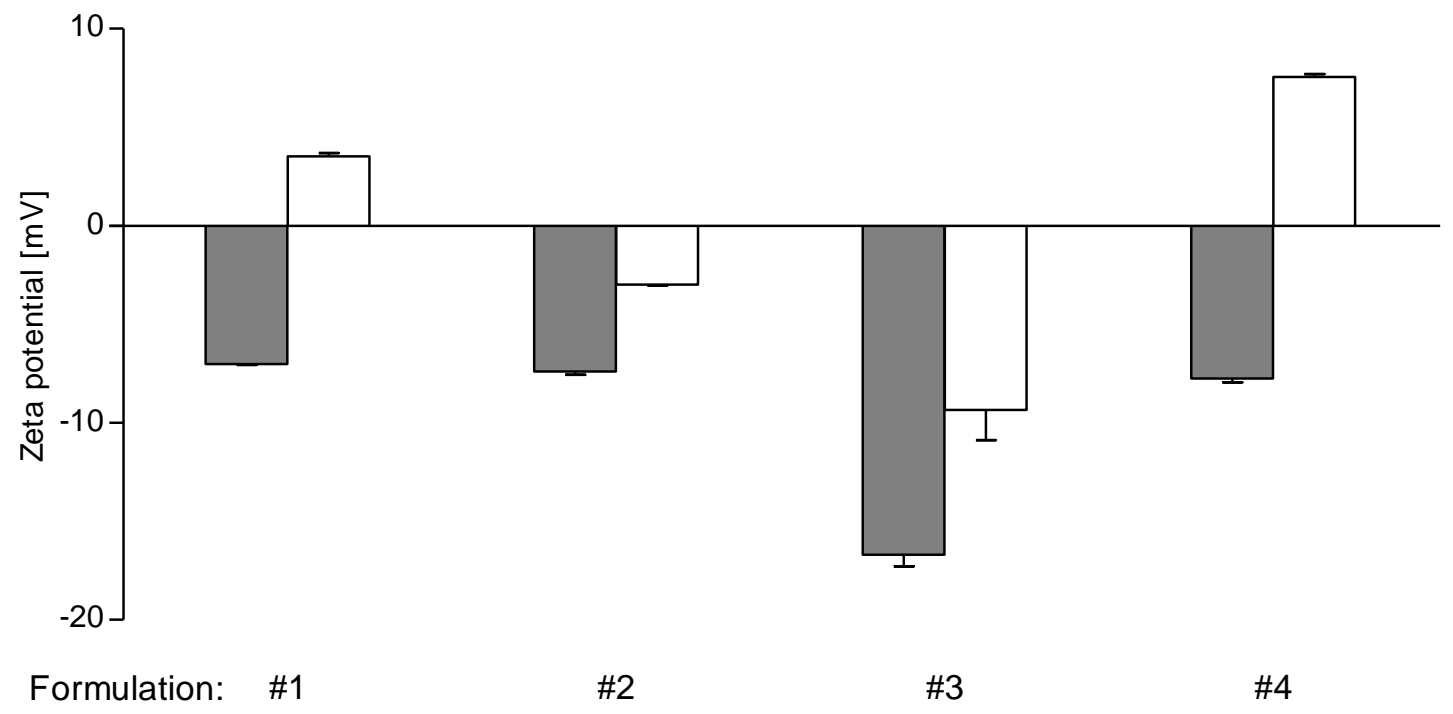

Change of $\zeta$ potential: $\zeta$ potential of different nanoparticle formulations before (gray bars) and after (white bars) enzymatic cleavage by IAP. Indicated values are the means of three independent experiments \pm standard deviation. The $\zeta$ potentials of each formulation before and after enzymatic cleavage differed significantly $(p<0.001)$. 


\section{Figure 3.}

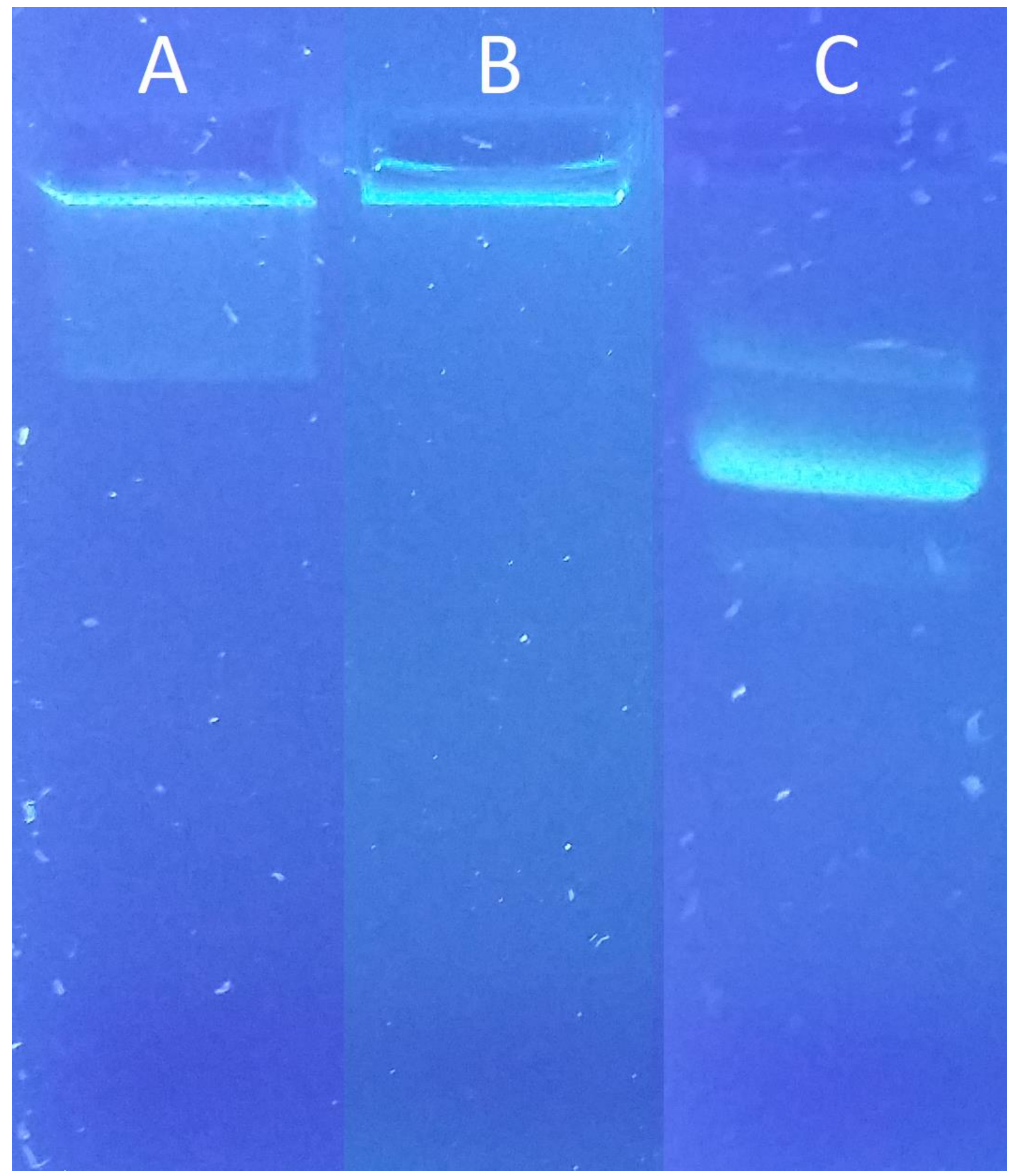

Encapuslation efficiency: agarose gel electrophoresis showing encapsulation of pDNA in formulation \#1 (A) and formulation \#4 (B). Naked pDNA (C) served as control. Gel was prestained with Midori Green Advance ${ }^{\circledR}$ and detected under UV light. 
Figure 4.
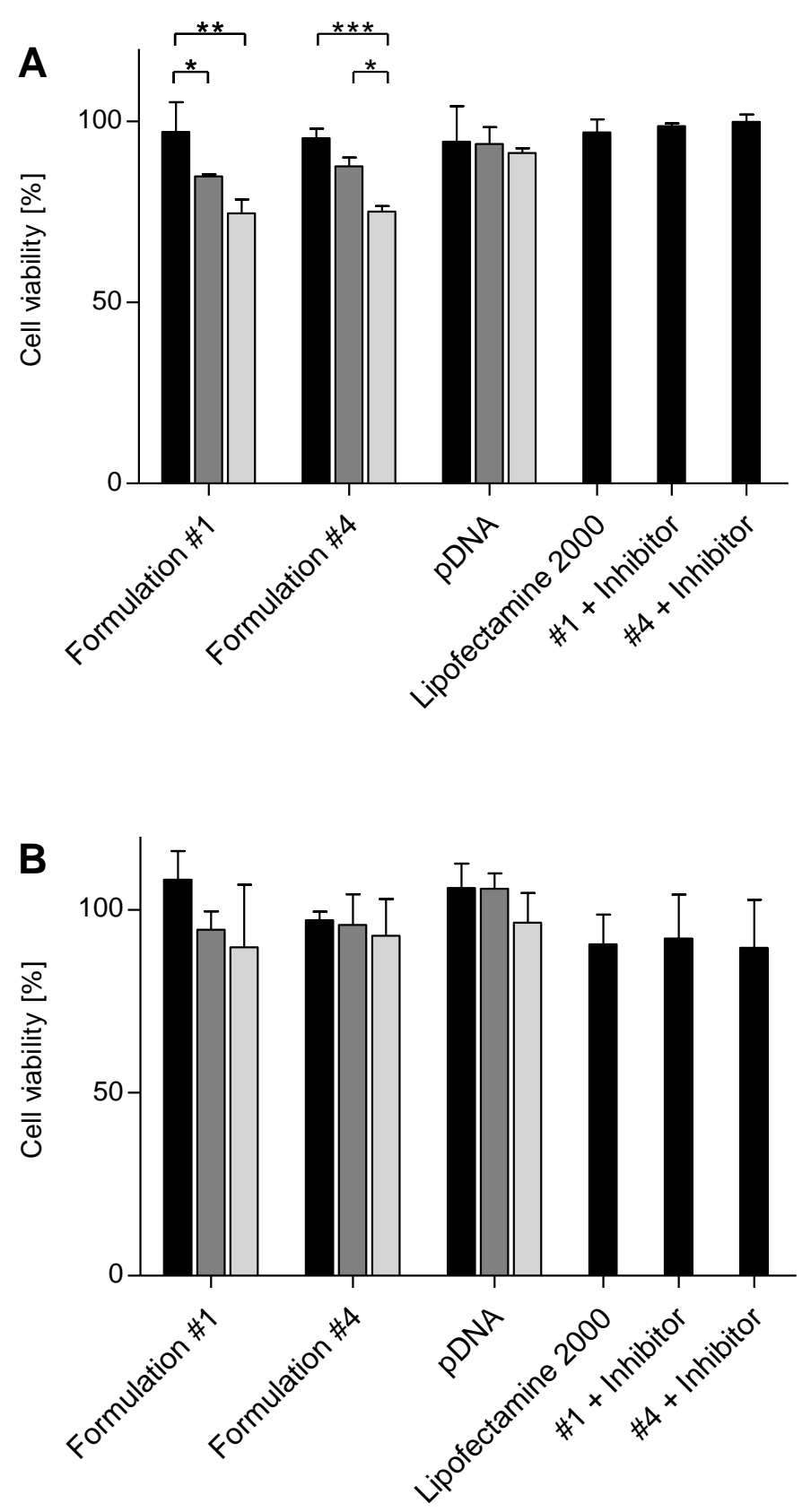

Cytotoxicity studies: Influence of formulations \#1 and \#4, naked pDNA and controls on cell viability of HEK-293 (A) and Caco-2 (B) cells by means of MTT assay after incubation of $4.5 \mathrm{~h}$ with corresponding final pDNA concentrations of 5 (black bars), 10 (gray bars) and 20 (white bars) $\mu \mathrm{g} / \mathrm{mL}$. Indicated values are the means of at least three independent experiments \pm standard deviation. Significant differences are indicated by $* p<0.05 ; * * p<0.001$. 
Figure 5.
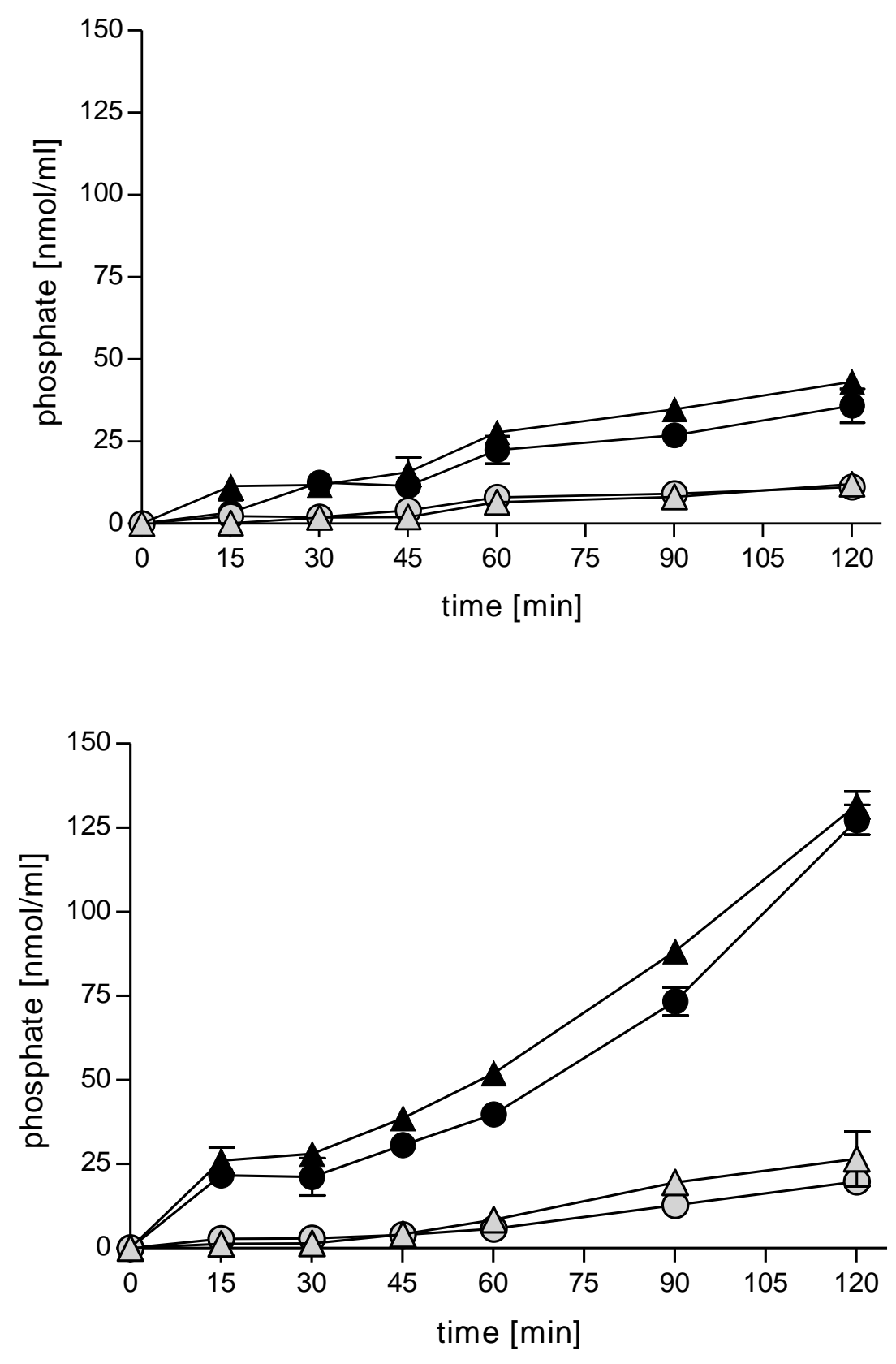

Time dependent phosphate release: Graph shows phosphate release from formulations \#1 (•) and \#4 ( $\mathbf{\Delta})$ and the controls with the addition of phosphatase inhibitor cocktail $(\# 1 \circ / \# 4 \Delta)$ after incubation on HEK-293 (A) and Caco-2 (B) cells. Indicated values are the means of at least three experiments \pm standard deviation. Indicated values are the means of at least three independent experiments \pm standard deviation. 
Figure 6.

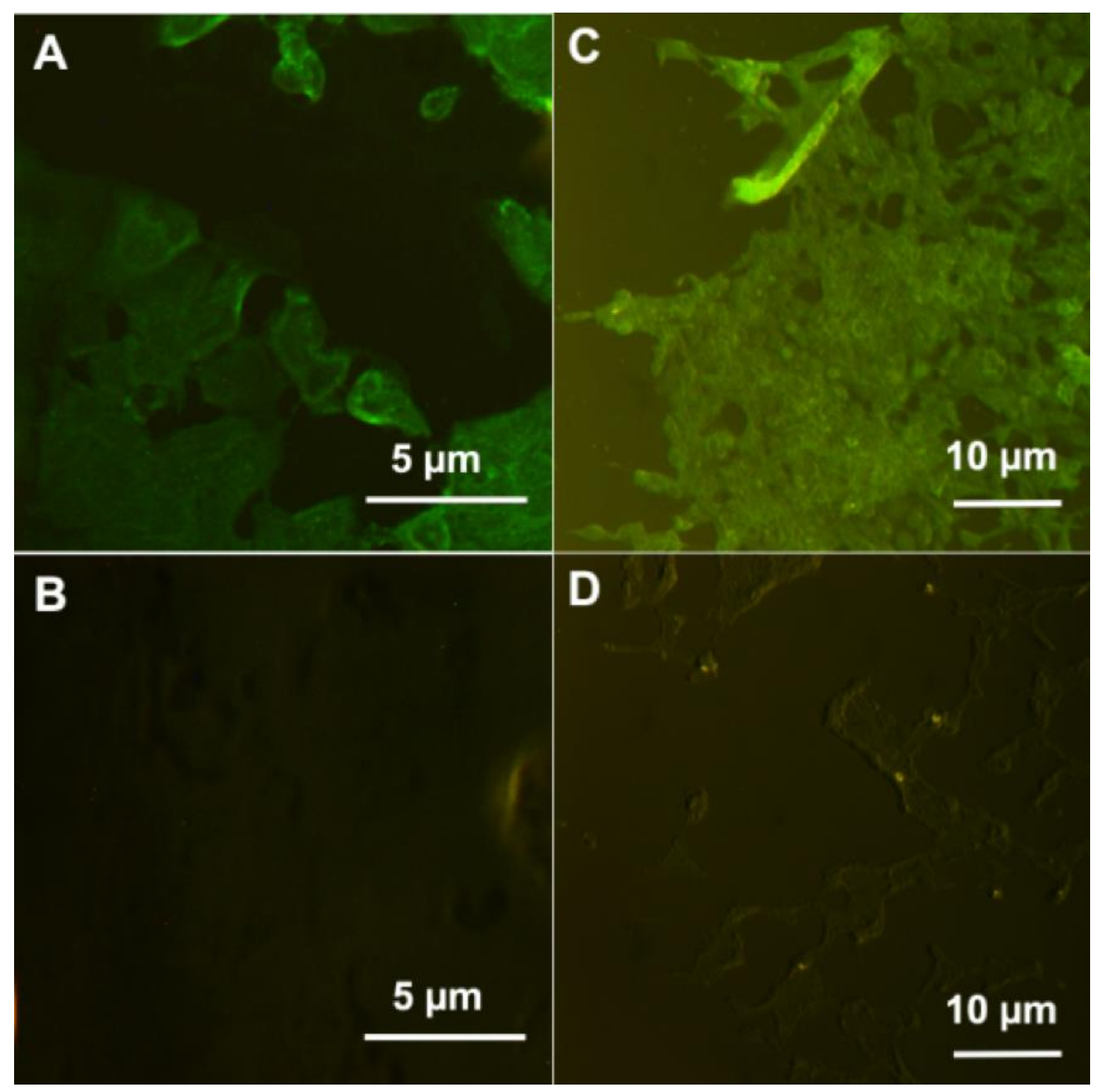

Immunocytochemistry images: stained alkaline phosphatase in HEK-293 cells (A: positive control and B: negative control) and in Caco-2 cells (C: positive control and D: negative control). 
Figure 7.
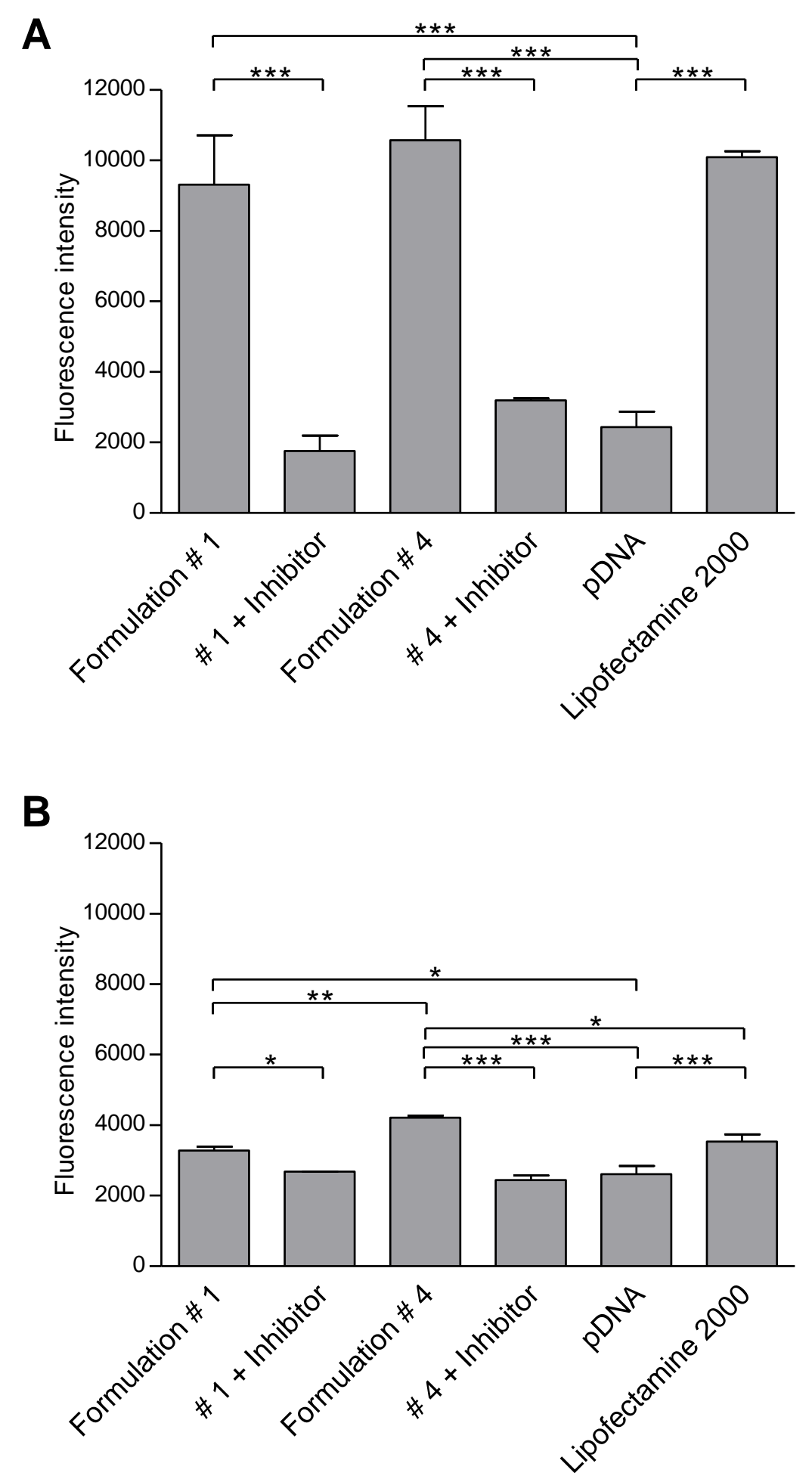

Transfection efficiency of formulations \#1 and \#4 and controls in HEK-293 (A) and Caco-2 (B) cells depicted as fluorescence intensity of GFP. The experiment was also carried out in the 
presence of a phosphatase inhibitor as control. Indicated values are the means of at least three independent experiments \pm standard deviation. Significant differences are indicated as ${ }^{*} p<0.05 ;{ }^{* *} p<0.01 ;{ }^{* *} p<0.001$. Fluorescence intensity of formulation \#1, formulation \#4 and Lipofectamine 2000 differed significantly from pDNA. Each formulation differed significantly from the controls with phosphate inhibitor. 
Table 1.

Composition of different nanoparticle formulations.

\begin{tabular}{cccc}
\hline Formulation & $\begin{array}{c}\text { Amount CS-PTyr } \\
(\mu \mathrm{g} / \mathrm{mL})\end{array}$ & $\begin{array}{c}\text { Amount pDNA } \\
(\mu \mathrm{g} / \mathrm{mL})\end{array}$ & $\begin{array}{c}\text { Amount CMC-PTyr } \\
(\mu \mathrm{g} / \mathrm{mL})\end{array}$ \\
\hline$\# 1$ & 100 & 65 & - \\
$\# 2$ & 100 & 75 & - \\
$\# 3$ & 100 & 85 & - \\
$\# 4$ & 100 & 65 & 200 \\
\hline
\end{tabular}

CMC: Carboxymethyl cellulose; CS: Chitosan; CS-PTyr: Phosphotyrosine-bearing CS derivative; CMC-PTyr: Phosphotyrosine-bearing CMC derivative; PTyr: Phosphotyrosine. 